\title{
Cooperative Shear Model for the Rheology of Glass-Forming Metallic Liquids
}

\author{
Marios D. Demetriou, ${ }^{1, *}$ John S. Harmon, ${ }^{1}$ Min Tao, ${ }^{2}$ Gang Duan, ${ }^{1}$ Konrad Samwer, ${ }^{3}$ and William L. Johnson ${ }^{1}$ \\ ${ }^{1}$ Keck Engineering Laboratories, California Institute of Technology, Pasadena, California 91125, USA \\ ${ }^{2}$ Graduate Aeronautical Laboratories, California Institute of Technology, Pasadena, California 91125, USA \\ ${ }^{3}$ I. Physik Institute, University of Goettingen, Goettingen, Germany \\ (Received 16 March 2006; published 11 August 2006; corrected 14 August 2006)
}

\begin{abstract}
A rheological law based on the concept of cooperatively sheared flow zones is presented, in which the effective thermodynamic state variable controlling flow is identified to be the isoconfigurational shear modulus of the liquid. The law captures Newtonian as well as non-Newtonian viscosity data for glassforming metallic liquids over a broad range of fragility. Acoustic measurements on specimens deformed at a constant strain rate correlate well with the measured steady-state viscosities, hence verifying that viscosity has a unique functional relationship with the isoconfigurational shear modulus.
\end{abstract}

DOI: 10.1103/PhysRevLett.97.065502

PACS numbers: 61.43.Fs, 61.66.Dk

Over the last three decades, several phenomenological theories have been proposed to explain flow in metallic glasses, most of which were founded on two hypothetical flow mechanisms: dilatation [1] and cooperative shear [2]. By analogy to granular materials, metallic glasses were thought to flow by deformation-induced dilatation, which results in the creation of a microstructural "free volume" leading to flow localization and consequent softening [1]. Owing to their ability to effectively capture the flow characteristics of metallic glasses, free volume models have been regarded as good phenomenological flow models and have been widely embraced. Even though experimental assessment of excess molar volume provided certain evidence of deformation-induced dilatation $[3,4]$, it has not been possible to quantitatively link measurable free volume to flow as predicted by free volume models. To some extent, this can be attributed to the lack of a fundamental thermodynamic definition of "free volume" leading to constitutive models that possibly lack thermodynamic consistency. In an alternative approach [2], flow in amorphous metals was thought to be accommodated by cooperative shearing of atomic clusters, referred to as "shear transformation zones." In a recent study [5], it has been shown that plastic yielding in metallic glasses can be effectively accounted for by adopting a cooperative yielding analysis for these flow zones similar to the one developed by Frenkel [6] for dislocation-free crystals. In the present study, we employ such cooperative shear flow analysis to investigate the rheology of metallic glass-forming liquids.

Following [5], a periodic energy density $\phi$ versus strain $\gamma$ can be formulated as $\phi / \phi_{0}=\sin ^{2}\left(\pi \gamma / 4 \gamma_{c}\right)$, where $\phi_{0}$ is the barrier energy density, and $\gamma_{c}$ is a critical shear strain limit shown to be a universal scale for metallic glasses. Considering that the shear modulus is given by the curvature of the energy density function, i.e., $G=$ $d^{2} \phi /\left.d \gamma^{2}\right|_{\gamma=0}$, a linear relationship between barrier energy density and shear modulus can be formulated as $\phi_{0}=$ $\left(8 / \pi^{2}\right) \gamma_{c}^{2} G$. Multiplying by an effective zone volume $\Omega$, the total energy barrier for configurational hopping be- tween inherent states, which can be regarded as the activation barrier for shear flow, can be expressed as $W=\left(8 / \pi^{2}\right) \gamma_{c}^{2} G \Omega$. Acknowledging that the variables contributing to barrier softening are $G$ and $\Omega$, the expression for the energy barrier can be rearranged as $W=$ $W_{0}\left(G / G_{0}\right)\left(\Omega / \Omega_{0}\right)$, where $G_{0}$ and $\Omega_{0}$ are characteristic scales for the shear modulus and the zone volume, and $W_{0} \equiv\left(8 / \pi^{2}\right) \gamma_{c}^{2} G_{0} \Omega_{0}$. Taking the barrier crossing rate normalized by an attempt frequency to follow a Boltzmann distribution function, we can arrive at a viscosity law based on barrier softening:

$$
\eta / \eta_{\infty}=\exp [W / k T]
$$

where $\eta_{\infty}$ is the Born-liquid limit of viscosity, which can be realized in the limit of $W \rightarrow 0$.

In the context of this analysis, Newtonian flow can be regarded as thermally activated flow where barriers are overcome entirely by thermal fluctuations. The viscosity should therefore be determined by the shear modulus and zone volume corresponding to the equilibrium configurational state, $G_{e}$ and $\Omega_{e}$, whose temperature dependence we describe by an exponential decay function, as $G_{e} / G_{0}=$ $\exp \left(-n T / T_{g}\right)$ and $\Omega_{e} / \Omega_{0}=\exp \left(-p T / T_{g}\right)$, where $T_{g}$ is the glass transition temperature. The form of this function originates from the probability distribution of inherent configurational states in a potential energy landscape model of a metallic glass [7]. In these expressions, $n$ and $p$ are indices quantifying the contributions of $G$ and $\Omega$ to the softening of $W$. The equilibrium barrier therefore takes the form $W_{e}=W_{0} \exp \left(-T / T_{w}\right)$, where $T_{w}=T_{g} /(n+p)$. Substituting into Eq. (1), an equilibrium viscosity law is obtained with two parameters, $W_{0}$ and $T_{w}$, as follows:

$$
\frac{\eta_{e}}{\eta_{\infty}}=\exp \left[\frac{W_{0}}{k T} \exp \left(-\frac{T}{T_{w}}\right)\right]
$$

In Fig. 1, we present the fit of the equilibrium law to Newtonian viscosity data of metallic glass-forming liquids. The law effectively captures the Newtonian viscosity of $\mathrm{Zr}_{41.2} \mathrm{Ti}_{13.8} \mathrm{Ni}_{10} \mathrm{Cu}_{12.5} \mathrm{Be}_{22.5}$ [8] and $\mathrm{Pd}_{40} \mathrm{Ni}_{40} \mathrm{P}_{20}$ [9-11] 


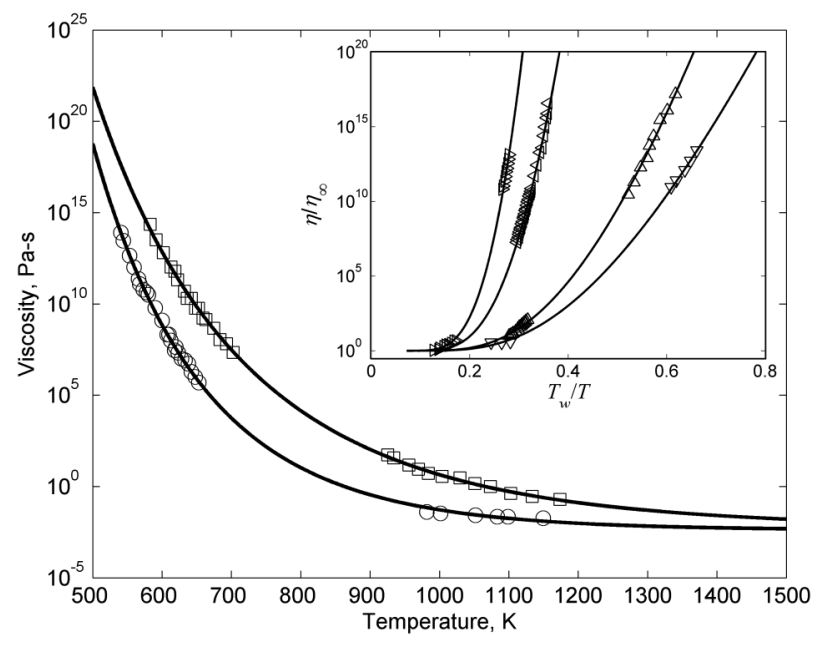

FIG. 1. Fit of the equilibrium viscosity law, Eqs. (1) and (2), to Newtonian data of metallic glass-forming liquids: $\mathrm{Zr}_{41.2} \mathrm{Ti}_{13.8} \mathrm{Ni}_{10} \mathrm{Cu}_{12.5} \mathrm{Be}_{22.5} \quad(\square) ; \quad \mathrm{Pd}_{40} \mathrm{Ni}_{40} \mathrm{P}_{20} \quad$ (○); $\mathrm{Pd}_{40} \mathrm{Ni}_{10} \mathrm{Cu}_{30} \mathrm{P}_{20}(\triangleleft) ; \mathrm{Pd}_{77.5} \mathrm{Cu}_{6} \mathrm{Si}_{16.5}(\triangleright) ; \mathrm{La}_{55} \mathrm{Al}_{25} \mathrm{Ni}_{20}(\nabla)$; $\mathrm{Mg}_{65} \mathrm{Cu}_{25} \mathrm{Y}_{10}(\triangle)$. Low-temperature viscosity data were produced by three-point beam bending, continuous-strain-rate tension and compression, and parallel-plate rheometry; hightemperature data were produced by concentric-cylinder rheometry, oscillating crucible, and electrostatic levitation [8$11,13,14,16-21]$.

over the entire range of temperatures studied rheologically. Moreover, plotted on a normalized plot [12] (insert in Fig. 1), the law captures Newtonian data of liquids ranging from the strongest to the most fragile [13,14,16-21], and can thus be perceived as a universal viscosity law. The fitting parameters are given in Table I. By comparison to free-volume based laws, the two-parameter softening law fits Newtonian data better than the two-parameter VogelFulcher-Tammann law [22], and at least as good as the three-parameter Cohen-Grest law [23].

As evidenced from Fig. 1 and Table I, fragile liquids are characterized by high $T_{g} / T_{w}$ ratios, which suggests that liquid fragility is dictated by $n+p$, i.e., by the combined softening effects of $G$ and $\Omega$. Since $G$ is determined by the morphology of the potential energy landscape (i.e., $\phi(\gamma)$ ), $n$ is expected to be unique for a given liquid. On the other hand, $\Omega$ is expected to be weakly dependent on the land- scape morphology and hence $p$ is expected to be similar for all liquids. Using a Debye model, one can estimate $p \cong$ $2 / 3$ [24]. From the functional dependencies of $G$ and $\Omega$, the relationship $\left(G / G_{0}\right)=\left(\Omega / \Omega_{0}\right)^{n / p}$ can be recognized, which leads to a correlation between $G$ and $W$ as $\left(G / G_{0}\right)=\left(W / W_{0}\right)^{q}$, or more importantly, between $G$ and $\eta$ as:

$$
\frac{G}{G_{0}}=\left[\frac{k T}{W_{0}} \ln \left(\frac{\eta}{\eta_{\infty}}\right)\right]^{q},
$$

where $q=n /(n+p)=1-(2 / 3)\left(T_{w} / T_{g}\right)$ and $G_{0}=$ $G_{e}\left(T_{g}\right) \exp \left(q T_{g} / T_{w}\right)$. Equation (3) essentially states that variations in viscosity correlate uniquely to variations in shear modulus.

We can now proceed to extend the softening law to the case of a driven system. A non-Newtonian flow law can be formulated by considering the flow-induced shift in the specific configurational potential energy of shear zones, $\varepsilon$, and its effect on $W$. The rate of barrier softening can thus be formulated as $\dot{W}_{\text {sof }}=\dot{\varepsilon} \delta W / \delta \varepsilon$, where $\dot{\varepsilon} \propto \eta \dot{\gamma}^{2}$ is the rate of production of specific configurational potential energy (taken to be proportional to the rate of dissipated energy density), $\dot{\gamma}$ is strain rate, and $\delta W / \delta \varepsilon=$ $(\partial W / \partial T) /(\partial \varepsilon / \partial T)$ is a thermodynamic parameter denoting changes in $W$ with respect to changes in $\varepsilon$. Near $T_{g}, \partial W / \partial T \approx-W_{0} \exp \left(-T_{g} / T_{w}\right) / T_{w}$ and $\partial \varepsilon / \partial T \approx$ $\left.(\partial h / \partial T)\right|_{T_{g}}$, where $h$ is the specific configurational enthalpy and $\left.(\partial h / \partial T)\right|_{T_{g}}$ can be evaluated from enthalpy recovery experiments. Configurational relaxation can be accounted for by adopting a unimolecular kinetic model as $\quad \dot{W}_{\text {rel }} \propto\left(W-W_{e}\right) / \tau_{M}$, where $\tau_{M}=\eta / G=$ $\eta /\left[G_{0}\left(W / W_{0}\right)^{q}\right]$ is the Maxwell relaxation time. Requiring $\dot{W}_{\text {sof }}=\dot{W}_{\text {rel }}$ for steady flow, we arrive at a self-consistent nonequilibrium law:

$$
\alpha \eta \dot{\gamma}^{2} \delta W / \delta \varepsilon=\frac{\left(W-W_{e}\right)\left(W / W_{0}\right)^{q}}{\eta / G_{0}} .
$$

In the above equation, $\alpha$ is a model parameter incorporating two unknown proportionality constants: the conversion efficiency of dissipated energy into potential energy, and a factor quantifying the deviation of the system's relaxation rate from Maxwellian.

TABLE I. Fit parameters to the equilibrium viscosity law, Eq. (1), for various metallic glass-forming liquids. It is noted that $\eta_{\infty}$ was assigned a value near the Planck limit which resulted in a best fit (as typically implemented when fitting viscosity).

\begin{tabular}{lccccc}
\hline \hline & $T_{g}[\mathrm{~K}]$ & $T_{w}[\mathrm{~K}]$ & $T_{g} / T_{w}$ & $W_{0}[\mathrm{~J}]$ & $\eta_{\infty}[\mathrm{Pa}-\mathrm{s}]$ \\
\hline $\mathrm{Zr}_{41.2} \mathrm{Ti}_{13.8} \mathrm{Ni}_{10} \mathrm{Cu}_{12.5} \mathrm{Be}_{22.5}[8]$ & 613 & 350 & 1.75 & $1.6 \times 10^{-18}$ & $5.6 \times 10^{-3}$ \\
$\mathrm{Pd}_{40} \mathrm{Ni}_{40} \mathrm{P}_{20}[9-11]$ & 560 & 222 & 2.52 & $3.2 \times 10^{-18}$ & $4.0 \times 10^{-3}$ \\
$\mathrm{Pd}_{43} \mathrm{Ni}_{10} \mathrm{Cu}_{27} \mathrm{P}_{20}[13,14]$ & 569 & 200 & 2.85 & $4.6 \times 10^{-18}$ & $2.2 \times 10^{-3}$ \\
$\mathrm{Pd}_{77.5} \mathrm{Cu}_{6} \mathrm{Si}_{16.5}[16,17]$ & 635 & 180 & 3.53 & $9.6 \times 10^{-18}$ & $1.0 \times 10^{-2}$ \\
$\mathrm{La}_{55} \mathrm{Al}_{25} \mathrm{Ni}_{2 .}[18,19]$ & 450 & 314 & 1.43 & $9.2 \times 10^{-19}$ & $5.3 \times 10^{-4}$ \\
$\mathrm{Mg}_{65} \mathrm{Cu}_{25} \mathrm{Y}_{10}[20,21]$ & 405 & 236 & 1.72 & $1.1 \times 10^{-18}$ & $1.5 \times 10^{-3}$ \\
\hline \hline
\end{tabular}




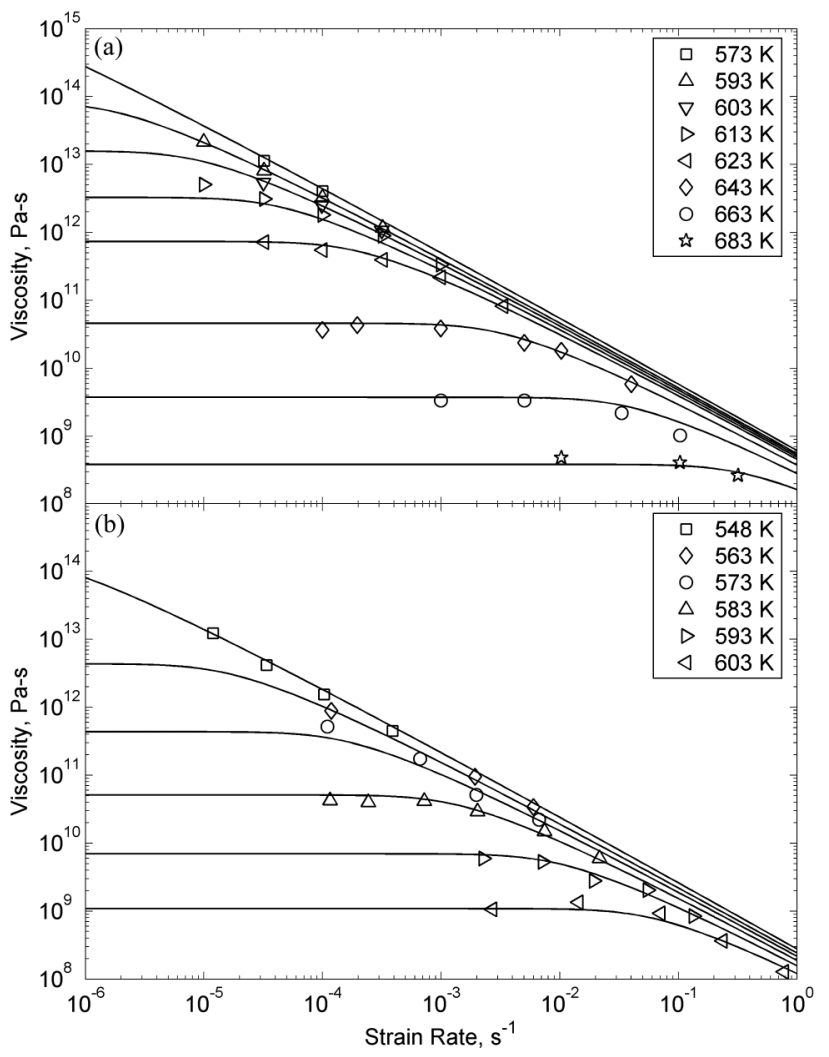

FIG. 2. Fit of the nonequilibrium viscosity law, Eq. (4), to the non-Newtonian data of (a) $\mathrm{Zr}_{41.2} \mathrm{Ti}_{13.8} \mathrm{Ni}_{10} \mathrm{Cu}_{12.5} \mathrm{Be}_{22.5}$ [15] and (b) $\mathrm{Pd}_{40} \mathrm{Ni}_{10} \mathrm{Cu}_{30} \mathrm{P}_{20}$ [14]. The data were obtained by continuous-strain-rate compression experiments using the Instron setup described in [15]. The small discrepancy in the Newtonian data of $\mathrm{Zr}_{41.2} \mathrm{Ti}_{13.8} \mathrm{Ni}_{10} \mathrm{Cu}_{12.5} \mathrm{Be}_{22.5}$ between $[8,15]$ was adjusted by introducing a temperature correction of $8 \mathrm{~K}$.

For $\mathrm{Zr}_{41.2} \mathrm{Ti}_{13.8} \mathrm{Ni}_{10} \mathrm{Cu}_{12.5} \mathrm{Be}_{22.5}, G_{e}\left(T_{g}\right) \approx 33 \mathrm{GPa}$ [25], and $\left.(\partial h / \partial T)\right|_{T_{g}} \approx 1.5 \mathrm{MJ} / \mathrm{m}^{3} \mathrm{~K} \quad[26]$ which gives $\delta W / \delta \varepsilon=-5.29 \times 10^{-28} \mathrm{~m}^{3}$. For $\mathrm{Pd}_{43} \mathrm{Ni}_{10} \mathrm{Cu}_{27} \mathrm{P}_{20}$, $G_{e}\left(T_{g}\right) \approx 31 \mathrm{GPa}$ [27], and $\left.(\partial h / \partial T)\right|_{T_{g}} \approx 2.5 \mathrm{MJ} / \mathrm{m}^{3} \mathrm{~K}$ [28] which gives $\delta W / \delta \varepsilon=-5.32 \times 10^{-28} \mathrm{~m}^{3}$. In Fig. 2 we present the solution of Eq. (4), superimposed on the non-Newtonian data of $\mathrm{Zr}_{41.2} \mathrm{Ti}_{13.8} \mathrm{Ni}_{10} \mathrm{Cu}_{12.5} \mathrm{Be}_{22.5}$ [15] and $\mathrm{Pd}_{43} \mathrm{Ni}_{10} \mathrm{Cu}_{27} \mathrm{P}_{20}$ [14], produced by adjusting $\alpha$ to 16 and 61 , respectively. Evidently, the nonequilibrium model seems capable of effectively capturing non-Newtonian viscosity data by adjustment of just one parameter.

We have therefore demonstrated that liquid fragility and strain-rate sensitivity are dictated by the softening of $W$ which is uniquely determined by the thermodynamics of $G$. We can therefore regard $G$ as the effective thermodynamic state variable governing flow. Contrary to free volume, which is presumed to vanish at some finite temperature below glass transition producing a singularity in viscosity, $G$ is thermodynamically well behaved rendering the viscosity law thermodynamically consistent. Fundamentally, $G$ represents the isoconfigurational shear modulus of the liquid at the high-frequency "solidlike" limit, and unlike free volume, is a thermodynamically well-defined and experimentally accessible property. Accordingly, acoustic measurements during mechanical deformation would be expected to correlate with viscosity assessed from measuring flow stress. We shall hence attempt to validate such correlation by measuring shear moduli of stressed configurational states and comparing them to the associated viscosities.

We utilized specimens of $\mathrm{Zr}_{41.2} \mathrm{Ti}_{13.8} \mathrm{Ni}_{10} \mathrm{Cu}_{12.5} \mathrm{Be}_{22.5}$ and $\mathrm{Pd}_{43} \mathrm{Ni}_{10} \mathrm{Cu}_{27} \mathrm{P}_{20}$ which have undergone mechanical deformation at constant rates at $593 \mathrm{~K}$ [15] and 548 K [14], respectively. Deformation was performed for sufficiently long time to allow a steady-state flow stress to be attained, and upon unloading, quenching was performed as rapidly as possible in an effort to freeze the configurational state associated with that flow stress. We evaluated the shear modulus of the quenched unloaded specimens using ultrasonic measurements along with density measurements [29]. Shear longitudinal wave speeds were measured using the pulse-echo overlap setup described in [25]. Densities were measured by the Archimedes method, as given in the American Society of Testing Materials standard C693-93. The room temperature measurements were corrected to estimate the shear modulus at the temperature of the flow experiment by accounting for the Debye-Grüneisen temperature effect on the shear modulus of the frozen glass. We utilized measured linear Debye-Grüneisen coefficients of $\sim 9 \mathrm{MPa} / \mathrm{K}$ for $\mathrm{Zr}_{41.2} \mathrm{Ti}_{13.8} \mathrm{Ni}_{10} \mathrm{Cu}_{12.5} \mathrm{Be}_{22.5}$ [25] and $\sim 15 \mathrm{MPa} / \mathrm{K}$ for $\mathrm{Pd}_{43} \mathrm{Ni}_{10} \mathrm{Cu}_{27} \mathrm{P}_{20}$ [27].

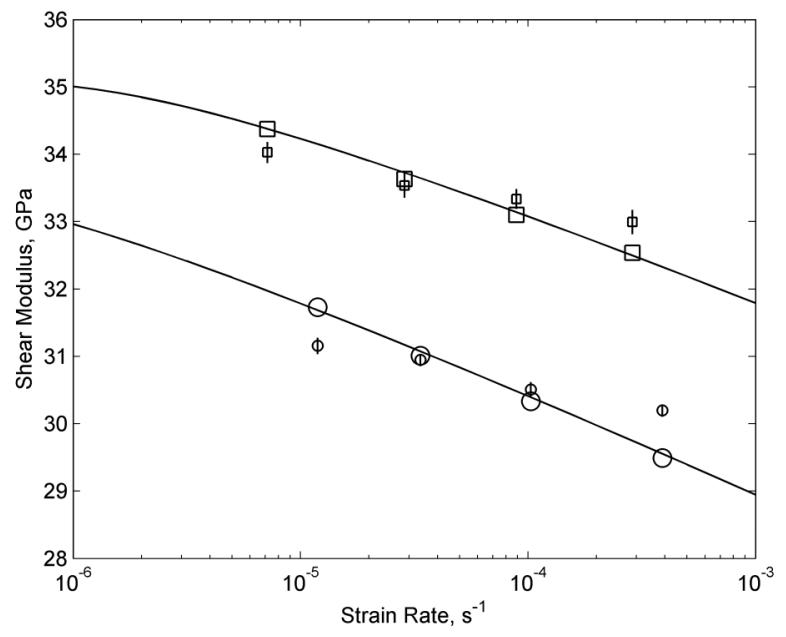

FIG. 3. Acoustically measured shear moduli (corrected for Debye-Grüneisen effect) of quenched unloaded specimens following steady deformation at the indicated rates: $\mathrm{Zr}_{41.2} \mathrm{Ti}_{13.8} \mathrm{Ni}_{10} \mathrm{Cu}_{12.5} \mathrm{Be}_{22.5}$ at $593 \mathrm{~K}(\square)$ and $\mathrm{Pd}_{40} \mathrm{Ni}_{40} \mathrm{P}_{20}$ at $548 \mathrm{~K}(\mathrm{O})$. Shear modulus predicted from viscosity data using Eq. (3): $\mathrm{Zr}_{41.2} \mathrm{Ti}_{13.8} \mathrm{Ni}_{10} \mathrm{Cu}_{12.5} \mathrm{Be}_{22.5}$ at $593 \mathrm{~K}$ ( $\square$ ) and $\mathrm{Pd}_{43} \mathrm{Ni}_{10} \mathrm{Cu}_{27} \mathrm{P}_{20}$ at $548 \mathrm{~K}(\mathrm{O})$. Solid lines are predictions from Eq. (4). 
The results from the acoustic measurements are presented in Fig. 3. The measurements clearly indicate the effect of strain rate on shear modulus. This effect has also been observed in recent molecular dynamics simulations [30]. In Fig. 3 we superimpose the shear moduli predicted from viscosities using Eq. (3), along with the solution of the nonequilibrium law, Eq. (4). As evidenced from Fig. 3, the shear modulus measured acoustically can be adequately correlated to the viscosity assessed from measuring flow stress. The small apparent discrepancy in this correlation may be related to the ex situ nature of the acoustic experiment. During unloading and quenching of specimens prior to measuring sound velocities, some degree of relaxation towards equilibrium might occur, or some fraction of potential energy might instantaneously recover as elastic, resulting in lower apparent strain-rate sensitivity.

In conclusion, we presented a rheological law based on the concept of cooperatively sheared flow zones, in which the effective thermodynamic variable governing flow is identified to be the isoconfigurational shear modulus of the liquid. We found the law capable of explaining the equilibrium as well as the nonequilibrium flow of metallic glass-forming liquids. We further demonstrated that variations in viscosity with both temperature and strain rate can be uniquely correlated to variations in isoconfigurational shear modulus, and hence verified that viscosity has a unique functional relationship and a one-to-one correspondence with shear modulus.

This work was supported by the MRSEC Program of the National Science Foundation under Grant No. DMR0520565. The authors are grateful to G. Ravichandran for providing the Instron apparatus and to M.L. Lind for providing the pulse-echo overlap setup.

*Author to whom correspondence should be addressed. Email address: marios@caltech.edu

[1] F. Spaepen, Acta Metall. 25, 407 (1977).

[2] A. S. Argon, Acta Metall. 27, 47 (1979).

[3] K. M. Flores, D. Suh, and R.H. Dauskardt, P. AsokaKumar, P. A. Sterne, and R. H. Howell, J. Mater. Res. 17, 1153 (2002).

[4] K. Hajlaoui, T. Benameur, G. Vaughan, and A. R. Yavari, Scr. Mater. 51, 843 (2004).

[5] W. L. Johnson and K. Samwer, Phys. Rev. Lett. 95, 195501 (2005).

[6] J. Frenkel, Z. Phys. 37, 572 (1926).

[7] W. L. Johnson (to be published).
[8] A. Masuhr, T. A. Waniuk, R. Busch, and W. L. Johnson, Phys. Rev. Lett. 82, 2290 (1999).

[9] K. H. Tsang, S. K. Lee, and H. W. Kui, J. Appl. Phys. 70, 4837 (1991).

[10] G. Wilde, G. P. Görler, K. Jeropoulos, R. Willnecker, and H. J. Fecht, Mater. Sci. Forum 269-272, 541 (1998).

[11] Y. Kawamura and A. Inoue, Appl. Phys. Lett. 77, 1114 (2000).

[12] C. A. Angell, Science 267, 1924 (1995).

[13] G. H. Fan, H.-J. Fecht, and E. J. Lavernia, Appl. Phys. Lett. 84, 487 (2004).

[14] Continuous-strain-rate compression tests were performed using the setup described in [15]. The specimens were prepared by first fluxing $\mathrm{Pd}_{43} \mathrm{Ni}_{10} \mathrm{Cu}_{27} \mathrm{P}_{20}$ alloy with $\mathrm{B}_{2} \mathrm{O}_{3}$ and subsequently casting into 4-mm diameter rods, whose amorphous nature was verified by differential scanning calorimetry. The rods were cut and polished to form 6-mm tall cylindrical specimens.

[15] J. Lu, G. Ravichandran, and W. L. Johnson, Acta Mater. 51, 3429 (2003).

[16] H. S. Chen, J. Non-Cryst. Solids 27, 257 (1978).

[17] I. Egry, G. Lohofer, I. Seyhan, S. Schneider, and B. Feuerbacher, Int. J. Thermophys. 20, 1005 (1999).

[18] Y. Kawamura, T. Nakamura, H. Kato, H. Mano, and A. Inoue, Mater. Sci. Eng. A 304-306, 674 (2001).

[19] T. Yamasaki, T. Tatibana, Y. Ogino, and A. Inoue, in Bulk Metallic Glasses, Proceedings of the 1998 MRS Fall Meeting, edited by W. L. Johnson, C. T. Liu, and A. Inoue (Materials Research Society, Pittsburgh, 1999), p. 63.

[20] S.-S. Wu, T.-S. Chin, K.-C. Su, and F.-H. Shyr, Jpn. J. Appl. Phys. 35, 175 (1996).

[21] R. Busch, W. Liu, and W. L. Johnson, J. Appl. Phys. 83, 4134 (1998).

[22] H. Vogel, Z. Phys. 22, 645 (1921); G. S. Fulcher, Am. Ceram. Soc. Bull. 8, 339 (1925); G. Tammann and G. Hesse, Z. Anorg. Allg. Chem. 156, 245 (1926).

[23] M.H. Cohen and G. S. Grest, Phys. Rev. B 20, 1077 (1979).

[24] W. L. Johnson (to be published).

[25] M. L. Lind, G. Duan, and W. L. Johnson, Phys. Rev. Lett. 97, 015501 (2006).

[26] R. Busch, Y. J. Kim, and W. L. Johnson, J. Appl. Phys. 77, 4039 (1995).

[27] N. Nishiyama, A. Inoue, and J. Z. Jiang, Appl. Phys. Lett. 78, 1985 (2001).

[28] X. Hu, Y. Li, S.C. Ng, and Y. P. Feng, Phys. Rev. B 62, 3169 (2000).

[29] E. Screiber, O. Anderson, and N. Soga, Elastic Constants and their Measurement (McGraw-Hill, New York, 1973), 1 st ed.

[30] M. Zink, K. Samwer, W. L. Johnson, and S. G. Meyer, Phys. Rev. B 74, 012201 (2006). 